\title{
Estilos de liderazgo en centros educativos de Heredia: un estudio comparativo entre el colectivo directivo y docente
}

\author{
Leadership styles in Heredia's educational institutions: a comparative \\ study among management and teaching staff \\ Estilos de liderança em centros educacionais de Heredia: um estudo \\ comparativo entre a administração e corpo docente
}

\author{
José Antonio García-Martínez \\ Universidad Nacional \\ Heredia, Costa Rica \\ jose.garcia.martinez@una.cr \\ (D) http://orcid.org/0000-0003-0709-0814 \\ Virginia Cerdas-Montano \\ Universidad Nacional \\ Heredia, Costa Rica \\ norma.cerdas.montano@una.cr \\ http://orcid.org/0000-0003-1705-4630
}

Recibido - Received - Recebido: 07/08/2020 Corregido - Revised - Revisado: 10/11/2020 Aceptado - Accepted - Aprovado: 20/11/2020

DOI: https://doi.org/10.22458/ie.v22i33.3081

URL: https://revistas.uned.ac.cr/index.php/innovaciones/article/view/3081

\begin{abstract}
Resumen: El liderazgo es una característica fundamental en las instituciones en general y en el ámbito educativo en particular. El estilo de liderazgo puede influir directamente en el clima organizacional de las instituciones, promoviendo un buen desempeño tanto del personal docente y administrativo como del colectivo estudiantil. La presente investigación responde a una metodología cuantitativa, con un diseño ex post facto y transversal, con la aplicación del cuestionario Multifactor Leadership Questionnaire (MLQ) a una muestra probabilística estratificada de 125 personas directivas y 584 docentes de centros educativos públicos de la Dirección Regional de Educación de Heredia. Los principales resultados muestran altas puntuaciones en los estilos de liderazgo transformacional y transaccional y bajas en el estilo laissez faire. Igualmente, se han detectado diferencias significativas entre la autopercepción de personas directoras y docentes en relación con los estilos mencionados. Aspectos relacionados con las tareas, creencias, confianza, formas de enfrentar los problemas, se destacan con percepciones diferentes entre ambos grupos de profesionales. La disociación sobre una misma realidad puede interferir en la gestión que realiza la persona directora para el cumplimiento de metas y objetivos comunes. La legitimación de la comunidad educativa hacia el liderazgo ejercido por el personal directivo es elemento clave para una gestión efectiva de la organización educativa.
\end{abstract}


Palabras clave: Liderazgo, gestión educativa, administración de la educación, personal educativo.

Abstract: Leadership is a fundamental feature of institutions in general and the field of education in particular. The leadership style can directly influence the organizational climate of the institutions, promoting a good performance in the administrative and teaching staff, as well as in the students. A quantitative methodology was used in the present research, with an ex-post-facto and cross-sectional design, applying the Multifactor Leadership Questionnaire (MLQ) to a stratified probabilistic sample of 125 managers and 584 teachers of public schools of the Regional Directorate of Education of Heredia. The main outcomes show high scores in the transformational and transactional leadership styles and low in the laissez-faire style. Likewise, significant differences have been detected between the self-perception of headteachers and teachers concerning these styles. Aspects related to tasks, beliefs, self-confidence, problem-solving set off with different perceptions between both groups of professionals. This dissociation from the same reality can interfere in the management carried out by school principals regarding common goal and objectives fulfillment. The legitimation of the educational community towards the leadership exercised by headteachers is a key element for effective educational organization management.

Keywords: Leadership, educational management, educational administration, educational personnel

Resumo: A liderança é uma característica fundamental nas instituições em geral e no campo educacional em particular; o estilo de liderança pode influenciar diretamente o clima organizacional das instituições, promovendo um bom desempenho tanto do pessoal docente, administrativo com do corpo estudantil. Esta pesquisa responde a uma metodologia quantitativa, com um desenho ex post facto e transversal, aplicando o Questionário de Liderança Multifatorial (MLQ - Multifactor Leadership Questionnaire) a uma amostra probabilística estratificada de 125 pessoas diretoras e 584 docentes de centros educacionais públicos da Direção Regional de Educação de Heredia. Os principais resultados mostram pontuações altas nos estilos de liderança transformacional e transacional e baixas no estilo laissez faire. Também foram detectadas diferenças significativas entre a autopercepção dos diretores e docentes em relação a estes estilos. Aspectos relacionados a tarefas, crenças, confiança, formas de enfrentar problemas, destacam-se com percepções diferentes entres os dois grupos de profissionais. Esta dissociação da mesma realidade pode interferir na gestão que realiza a pessoa diretora no cumprimento das metas e objetivos comuns. A legitimação da comunidade educativa em relação à liderança exercida pelo pessoal diretor é um elemento chave para uma gestão eficaz da organização educativa.

Palavras-chave: Liderança, gestão educacional, administração da educação, profissionais da educação. 


\section{INTRODUCCIÓN}

El liderazgo es un término utilizado de manera recurrente en las ciencias sociales, en los ámbitos empresariales, políticos y educativos, siendo el mismo eje de investigación para acercamientos conceptuales y prácticos (Perles y Santiago, 2000). Los estudios acerca de los estilos de liderazgo son vastos, tanto en el contexto nacional como internacional (García y Cerdas, 2019; García, Cerdas y Torres, 2018; Sánchez, 2015; Torrecilla, 2016; Vílchez, Alberola, Monte y Ferraz, 2019), se detallan elementos de análisis que permiten una mejor comprensión de la dinámica de gestión de los centros educativos y su vinculación directa con el liderazgo para un trabajo colaborativo y el logro de metas comunes.

Al respecto, destacan estudios sobre estilos de liderazgo, donde se aplica el cuestionario elaborado por Bass \& Avolio (1994) y denominado Multifactor Leadership Questionnaire (MLQ) en el ámbito educativo. Flores y Homrani (2015) aportan desde un estudio cuantitativo que el liderazgo transformacional contribuye de manera indirecta a la mejora de los resultados del proceso de enseñanza y aprendizaje en el estudiantado. Lo anterior, mediante la participación en la toma de decisiones, la motivación del cuerpo docente y la distribución del liderazgo que repercuten en las prácticas educativas adaptadas a diversos contextos socioculturales.

Dean, Rushton, Edwards \& Jarrell (2016) realizan hallazgos importantes desde un enfoque cuantitativo con el profesorado que inicia sus labores en educación, los cuales creen poseer las cualidades de liderazgo transformacional y transaccional, condición relevante de autopercepción para enfrentar los retos que surgen en la labor como principiantes.

En esta misma línea, Hauserman \& Stick, (2013), en un estudio mixto, destacan que el liderazgo transformacional es asociado por el colectivo docente con una cultura organizacional positiva en sus centros educativos y con expresiones de admiración y respeto hacia las personas directivas; sin embargo se identifican bajos niveles de liderazgo asociados con comportamientos negativos y de frustración para el desarrollo del centro educativo.

Los términos motivación, autoridad, sinergia y equipo son asociados a la conceptualización de liderazgo en el ámbito organizacional (Carreño, 2020). Los acercamientos desde perspectivas políticas, sociales y culturales contribuyen a que este concepto sea el foco de investigación del personal académico alrededor del mundo (Hurtado, 2019).

En el ámbito educativo, el liderazgo se vincula con los esfuerzos colectivos en relación con el desarrollo del currículo para alcanzar procesos de enseñanza y aprendizajes efectivos alineados con la oferta educativa (Contreras, 2016). En este mismo sentido, Bolívar (2015) refiere que el liderazgo pedagógico se vincula a la influencia de la persona directora para generar una visión común con miras al mejoramiento permanente del centro educativo, enfatizando en los procesos de aprendizaje (Cerdas, García, Torres y Vargas, 2017).

En esta misma línea, se asume el liderazgo pedagógico desde la labor que se ejerce en el ámbito educativo, impactando en el cuerpo docente y en el estudiantado (Rodríguez, 2011), y promoviendo procesos de aprendizaje que respondan a las necesidades de los contextos (Gajardo y Ulloa, 2016). Desde este marco de acción, se plantea el análisis en tres tipos de liderazgo: transaccional, transformacional y laissez-faire.

Para el ejercicio del liderazgo transformacional, es necesaria la comprensión integral de los entornos culturales, sociales y políticos que permitan avanzar hacia compromisos individuales y colectivos en la organización (Gil, Muñíz y Delgado, 2008). Se apunta hacia el cambio auténtico de sus miembros en función de la misión y la visión del centro educativo (Duitama, 2017), favoreciendo la proactividad, la crítica constructiva y una toma de conciencia dirigida el bien común (Barrios, 2020; Villa, 2019).

En el estilo transformacional se destacan algunos componentes básicos que caracterizan a las personas directoras que lo ejercen. La estimulación intelectual es un aspecto clave que permite al equipo generar 
soluciones creativas e innovadoras ante los problemas (López, 2017). En este sentido, se favorece la reflexión sobre sus creencias y las nuevas perspectivas para la búsqueda de soluciones conjuntas, por tanto, nuevas maneras para asumir las tareas de la organización. Por su parte, la influencia idealizada se divide en dos dimensiones: una asociada con la conducta y la otra con atributos de la persona directora. En el primer caso, se vincula la capacidad del líder para generar confianza y credibilidad en el equipo de trabajo y direccionar la organización hacia una misión conjunta en donde se trazan valores compartidos (López, 2017). En cuanto a la influencia idealizada por atributos, las personas líderes son admiradas, respetadas e imitadas por las personas que forman parte del equipo (Castro, 2015). Por otro lado, la motivación por inspiración es la capacidad de la persona líder de impregnar en el equipo ideas de optimismo y expectativas para mejorar y enfrentar de manera conjunta los problemas (Hoy y Miskel, 2008). Finalmente, las consideraciones individuales se asocian con los vínculos relacionales que ejerce la persona directora con cada uno de los miembros del equipo, destacando la motivación individual para alentarlos a desarrollar sus potencialidades (Perilla, 2015).

El liderazgo transaccional supone un modelo de intercambio entre la persona que lidera y las que forman parte del equipo (Espinoza y Elgoibar, 2019), el cual favorece capacidades individuales y basa su atención en la recompensa contingente (Fernández y Quintero, 2017; Orellan, 2019; Prieto, Contreras y Espinosa, 2019).

Entre los componentes que caracterizan el estilo transaccional se destacan el premio por contingencia, que según Brito (2016) es la recompensa del equipo por su esfuerzo. En este sentido, alcanzar los objetivos propuestos cumpliendo con las expectativas de rendimiento es motivo de reconocimiento y satisfacción. Otro componente es la gerencia por excepción, dividida en las dimensiones pasiva y activa. La primera refiere, según Brito (2016), a una intervención tardía de los problemas que aquejan la organización. Mientras que, en la gerencia por excepción activa, la persona que lidera interviene de manera constante en los procesos de la organización respondiendo a los errores o los aciertos (Orellana, 2019).

El estilo de liderazgo laissez-faire procura de manera intencionada la horizontalidad del poder y autoridad (Pacsi, Estrada, Pérez y Cruz 2015), aspecto que repercute en una organización que avanza por la voluntad de sus miembros, sin que exista un norte definido de metas comunes. Esto implica que los esfuerzos individuales se diluyen entre la burocracia administrativista, sin que nadie se responsabilice de manera directa de los logros o fracasos. (Campos y Campos, 2014; Yang, 2015; Zuzama, 2015).

En cuanto a las características del estilo laissez-faire, la responsabilidad queda delegada en las personas que componen la organización con ausencia de la persona que lidera los procesos, lo que repercute en que la solución a los problemas que aquejan a esta sea asumida por cada miembro del equipo (Zuzama, 2015).

Finalmente, el cuestionario MLQ valora tres componentes adicionales que no se adjudican a las características de los estilos de liderazgo analizados, pero que refieren a aspectos importantes de la organización: efectividad, esfuerzo extra y satisfacción. La efectividad parte de la capacidad de la persona líder para estimular el logro de objetivos con la menor utilización de recursos (Bracho y García, 2013). El esfuerzo extra plantea la disposición de los miembros del equipo para asumir retos y tareas en función de los objetivos de la organización (Ayoub, 2011). Por último, la satisfacción refiere a un clima organizacional saludable en donde sus miembros se sienten retribuidos y apoyan las decisiones de la persona que lidera la organización (García, Mendoza y Ruíz, 2015).

En el contexto educativo costarricense este estudio se destaca como pionero, y tiene por objetivo comparar la autopercepción del estilo de liderazgo entre el personal directivo y el cuerpo docente de los centros educativos de la Dirección Regional de Educación de Heredia (DREH). 


\section{MATERIALES Y MÉTODOS}

El estudio se llevó a cabo desde un enfoque cuantitativo y un alcance de carácter exploratorio y descriptivo. El diseño es no experimental, ya que no existe la manipulación de variables, y transaccional, dado que la recolección de datos se realizó en un solo momento (Hernández, Fernández y Baptista, 2010).

La población ( $\mathrm{N}=4953)$ queda compuesta por el personal docente y directivo de centros educativos públicos de la DREH. Al ser un estudio comparativo, la muestra total $(n=707)$ responde por un lado a las personas directivas $(n=125)$ de centros educativos $y$, por otro, al colectivo docente $(n=584)$ de dichos centros públicos de la DREH. La muestra se seleccionó de forma probabilística y estratificada (Hernández et al., 2010), considerando como estrato cada uno de los siete circuitos que componen la DREH.

Para el cálculo muestral de ambos colectivos, se utilizó la formulación para poblaciones finitas propuesta por Arnal, Rincón y Latorre (1992). Teniendo en cuenta la población total de directores y directoras $(\mathrm{N}=214)$ y del personal docente ( $\mathrm{N}=4953)$; un nivel de confianza $\geq 95 \%$, error $\leq 5 \%$ y con distribución estimada de $50 \%$ de las respuestas, se obtiene que el número de personas encuestadas no debe ser inferior a 105 en el caso del personal directivo y 357 en el personal docente, para que la selección quede justificada.

Del total de personas directoras $35,2 \%$ son hombres y el restante $64,8 \%$ son mujeres. La edad oscila entre los 32 y los 58 años ( $\bar{X}=24 ; \sigma=5,9)$. Mientras que, del colectivo docente, un $15,5 \%$ son hombres y el $84,5 \%$ mujeres. En este caso la edad varía en un rango de 21 a 64 años ( $\bar{X}=21 ; \sigma=15,8)$. Cabe destacar, que todas las personas pertenecientes a la muestra debían haber laborado al menos un año en la institución en el momento de la recolección de datos.

La recogida de datos se realizó a través de la técnica de encuesta, y como instrumento se utiliza el Multifactor Leadership Questionnaire (MLQ 5X) de Bass y Avolio (1994). El cuestionario consta de varias partes, la primera con preguntas cerradas sobre aspectos generales; la segunda parte acerca de aspectos laborales y de formación; por último, una escala tipo Likert compuesta de 45 ítems con cinco opciones de respuesta $(1=$ nunca) hasta $(5=$ siempre). La escala mide tres estilos de liderazgo: transformacional ( 20 ítems), transaccional (12 ítems) y laissez-faire (4 ítems), además de tres subescalas relacionadas con la efectividad (4 ítems), satisfacción (25 ítems) y esfuerzo extra (3 ítems).

EI MLQ ha sido validado por múltiples autores y en diferentes contextos. Al respecto, Sharma (2020) concluye que el modelo inicial con los tres estilos de liderazgo sigue siendo apropiado, de acuerdo con las cargas factoriales obtenidas. Igualmente, se realizó una prueba piloto con 10 personas directoras y 25 docentes con características similares a la muestra final, con el objetivo de comprobar el comportamiento de los ítems, aspecto que permitió mejorar la redacción de algunas afirmaciones. Por último, se realizó la prueba de consistencia interna Alpha de Cronbach, y se obtuvo un coeficiente de 0,74 para los directores y de 0,82 en el caso del personal docente, considerados fiables por la teoría y con resultados similares a estudios como el de Sharma (2020).

La aplicación del cuestionario se realizó, con ambas poblaciones, en formato impreso. En el caso del personal directivo, previa coordinación con la persona supervisora de cada uno de los siete circuitos. Para acceder al colectivo docente, se solicitó permiso a la DREH y, posteriormente, con la persona directiva de los diferentes centros públicos participantes en el estudio.

Cabe destacar, que el cuestionario incluye en la primera página una introducción, donde se indica el objetivo del estudio, los beneficios de su participación, el carácter voluntario donde se indica la posibilidad de abandonar la encuesta en cualquier momento sin explicaciones previas, se garantiza el anonimato y se explica el tratamiento de los datos. Antes de la aplicación se realiza una lectura y explicación de todos los aspectos mencionados, garantizando la comprensión y atendiendo posibles respuestas por parte del equipo investigador, la entrega del cuestionario completo implica el consentimiento informado. 
Una vez aplicados los cuestionarios se procedió a introducir los datos en una matriz elaborada con un paquete estadístico. Se realizaron distintos análisis: estadísticos descriptivos (media y desviación típica), tablas de contingencia para el cálculo del chi-cuadrado $\left(\chi^{2}\right)$ y la V de Cramer para identificar diferencias entre ambos colectivos descritos anteriormente y pruebas de comparación de medias (t de Student).

\section{RESULTADOS}

En relación con el estilo de liderazgo transformacional (tabla 1), se subrayan las altas puntuaciones obtenidas tanto desde la autopercepción del colectivo director como de docentes en cada uno de los componentes de esta variable, destacando los elevados valores de la media en motivación por inspiración.

El colectivo directivo obtiene puntuaciones más altas en todos los ítems que las que otorgadas al colectivo docente. Sin embargo, se han encontrado diferencias significativas en algunas de las afirmaciones: tal es el caso del ítem "sugiero nuevas formas de considerar la realización de las tareas", $\left(\chi^{2}=16,3 / p=, 000 ; V\right.$ de Cramer $=, 15$ ) perteneciente al componente estimulación intelectual. Igualmente, el ítem "hablo sobre mis creencias y valores más importantes" $\left(\chi^{2}=24,1 / p=, 000\right.$; $V$ de Cramer $\left.=, 18\right)$ perteneciente al componente influencia idealizada por conducta y "genero orgullo en los demás por relacionarse conmigo" $\left(\chi^{2}=\right.$ 13,9 / $p=, 008 ; \mathrm{V}$ de Cramer = ,14) de influencia idealizada por atributos. Del componente motivación por inspiración, se han encontrado diferencias en los ítems "hablo con entusiasmo de las cosas que deben llevarse a cabo" $\left(\chi^{2}=18,2\right.$ / $p=, 000 ; V$ de Cramer $\left.=, 16\right)$ y "manifiesto mi confianza de que se alcanzarán los objetivos" ( $\chi^{2}=13,9 / p=, 000 ; V$ de Cramer $\left.=, 14\right)$. En relación con el componente consideraciones individuales, se encuentran diferencias en tres ítems, "dedico tiempo a enseñar y a instruir" $\left(\chi^{2}=15,2\right.$ / $p=, 004 ; \mathrm{V}$ de $C r a m e r=, 15)$; "considero que cada individuo posee necesidades, aptitudes y aspiraciones distintas a los demás" $\left(\chi^{2}=17,7 / p=, 000 ; V\right.$ de Cramer $\left.=, 16\right)$ y "Ayudo a los demás a desarrollar sus puntos fuertes" ( $\chi^{2}=28,4 / p=, 000 ; \mathrm{V}$ de Cramer $\left.=, 20\right)$.

TABLA 1

Porcentajes de frecuencia y estadísticos del liderazgo transformacional

\begin{tabular}{|c|c|c|c|c|c|c|c|c|}
\hline Estimulación intelectual & Roll & 1 & 2 & 3 & 4 & 5 & $\mathrm{x}$ & DS \\
\hline \multirow{2}{*}{$\begin{array}{l}\text { 2. Reexamino asunciones de manera crítica para plantearme } \\
\text { si son las adecuadas. }\end{array}$} & $\mathrm{D}$ & 3,2 & 1,6 & 16,8 & 32,8 & 45,6 & 4,13 & 1,05 \\
\hline & $\mathrm{P}$ & 8,9 & 9,4 & 13,7 & 28,8 & 39,2 & 3,80 & 1,29 \\
\hline \multirow{2}{*}{ 8. Al resolver problemas busco diferentes perspectivas } & $\mathrm{D}$ & 2,4 & 1,6 & 2,4 & 20,8 & 72,8 & 4,60 & 0,82 \\
\hline & $\mathrm{P}$ & 3,6 & 5,1 & 9,8 & 26,7 & 54,8 & 4,24 & 1,05 \\
\hline \multirow{2}{*}{$\begin{array}{l}\text { 30. Hago que los demás enfoquen los problemas desde } \\
\text { muchos ángulos distintos. }\end{array}$} & $\mathrm{D}$ & 4,0 & 2,4 & 8,0 & 40,8 & 44,8 & 4,20 & 0,98 \\
\hline & $P$ & 5,7 & 8,8 & 13,5 & 34,8 & 37,2 & 3,89 & 1,16 \\
\hline \multirow{2}{*}{$\begin{array}{l}\text { 32. Sugiero nuevas formas de considerar la realización de } \\
\text { las tareas. }\end{array}$} & $\mathrm{D}$ & 8 & 8 & 5,6 & 33,6 & 59,2 & 4,50 & 0,71 \\
\hline & $\mathrm{P}$ & 2,1 & 7,4 & 13,0 & 30,4 & 47,1 & 4,13 & 1,03 \\
\hline \multicolumn{9}{|l|}{ Influencia idealizada (conducta) } \\
\hline \multirow{2}{*}{ 6. Hablo sobre mis creencias y valores más importantes } & $\mathrm{D}$ & 12,8 & 12,0 & 21,6 & 32,8 & 20,8 & 3,37 & 1,29 \\
\hline & $\mathrm{P}$ & 9,5 & 9,0 & 12,6 & 25,1 & 43,8 & 3,85 & 1,30 \\
\hline \multirow{2}{*}{$\begin{array}{l}\text { 14. Hago hincapié en la importancia de poseer una sólida } \\
\text { visión de propósito. }\end{array}$} & D & 1,6 &, 0 & 4,0 & 20,8 & 73,6 & 4,65 & 0,71 \\
\hline & $P$ & 1,6 & 4,5 & 8,4 & 20,4 & 65,1 & 4,43 & 94 \\
\hline \multirow{2}{*}{$\begin{array}{l}\text { 23. Considero las consecuencias morales y éticas de mis } \\
\text { decisiones. }\end{array}$} & $\mathrm{D}$ & ,8 & 8 & 8,0 & 23,2 & 67,2 & 4,55 & 0,74 \\
\hline & $P$ & 2,2 & 6,5 & 10,8 & 22,5 & 58,0 & 4,27 & 1,03 \\
\hline \multirow{2}{*}{$\begin{array}{l}\text { 24. Recalco la importancia de tener un sentido colectivo } \\
\text { de misión. }\end{array}$} & $D$ & 3,2 & 1,6 & 8,8 & 33,6 & 52,8 & 4,23 & 1,26 \\
\hline & $\mathrm{P}$ & 3,6 & 7,9 & 14,8 & 32,4 & 41,3 & 4,00 & 1,09 \\
\hline
\end{tabular}




\section{Influencia idealizada (atributos)}

10. Genero orgullo en los demás por relacionarse conmigo.

\begin{tabular}{|r|r|r|r|r|r|r|r|}
\hline X & 20,8 & 7,2 & 16,8 & 20,8 & 34,4 & 4,12 & 1,08 \\
\hline P & 13,8 & 13,3 & 10,3 & 23,6 & 39,0 & 3,61 & 1,45 \\
\hline D & 12,8 & 7,2 & 9,6 & 25,6 & 44,8 & 3,82 & 1,40 \\
\hline P & 13,0 & 11,9 & 13,3 & 26,9 & 34,9 & 3,59 & 1,40 \\
\hline D & 4,8 & 3,2 & 4,8 & 27,2 & 60,0 & 4,34 & 1,05 \\
\hline P & 4,1 & 5,0 & 8,2 & 22,6 & 60,1 & 4,30 & 1,08 \\
\hline D & 29,6 & 13,6 & 16,0 & 20,8 & 20,0 & 2,88 & 1,53 \\
\hline P & 23,8 & 16,4 & 13,2 & 21,6 & 25,0 & 3,08 & 1,52 \\
\hline
\end{tabular}

\section{Motivación por inspiración}

9. Soy optimista cuando hablo del futuro.

\begin{tabular}{|r|r|r|r|}
\hline D & 1,6 &, 0 & 4,0 \\
\hline P & 2,1 & 2,2 & 6,0 \\
\hline D & 3,2 &, 8 & \\
\hline P & 2,2 & 4,8 & 7,0 \\
\hline D & 2,4 &, 8 & 8,8 \\
\hline P & 4,3 & 4,6 & 9,8 \\
\hline D &, 0 &, 0 & \\
\hline P & 2,6 & 3,8 & 9,0 \\
\hline
\end{tabular}

\begin{tabular}{|r|r|r|r|r|}
\hline 4,0 & 24,0 & 70,4 & 4,62 & 0,72 \\
\hline 6,0 & 19,5 & 70,2 & 4,54 &, 866 \\
\hline 0 & 13,6 & 82,4 & 4,71 & 0,80 \\
\hline 7,1 & 18,8 & 67,1 & 4,44 &, 97 \\
\hline 8,8 & 33,6 & 54,4 & 4,37 & 0,87 \\
\hline 9,8 & 23,8 & 57,5 & 4,46 & 1,08 \\
\hline, 8 & 12,8 & 86,4 & 4,86 & 0,37 \\
\hline 9,0 & 23,1 & 61,5 & 4,37 &, 97 \\
\hline
\end{tabular}

\section{Consideraciones individuales}

15. Dedico tiempo a enseñar y a instruir.

19. Trato a los demás cómo individuos más que como miembros del grupo.

29. Considero que cada individuo posee necesidades, aptitudes y aspiraciones distintas a los demás.

31. Ayudo a los demás a desarrollar sus puntos fuertes.

\begin{tabular}{|r|r|r|r|r|r|r|r|}
\hline D &, 0 &, 8 & 7,2 & 28,0 & 64,0 & 4,55 & 0,66 \\
\hline P & 3,8 & 7,5 & 8,9 & 37,9 & 51,9 & 4,17 & 1,10 \\
\hline D & 17,6 & 8,0 & 11,2 & 22,4 & 40,8 & 3,61 & 1,51 \\
\hline P & 15,4 & 13,7 & 11,3 & 19,4 & 40,2 & 3,55 & 1,50 \\
\hline D & 6,4 &, 0 & 4,8 & 11,2 & 77,6 & 4,54 & 1,06 \\
\hline P & 2,6 & 3,9 & 9,2 & 18,8 & 65,5 & 4,41 &, 98 \\
\hline D &, 0 &, 8 & 5,6 & 20,8 & 72,8 & 4,66 & 0,62 \\
\hline P & 2,9 & 7,0 & 12,7 & 28,6 & 48,8 & 4,13 & 1,06 \\
\hline
\end{tabular}

Fuente: Elaboración propia.

Notas: $n=709$ (125 directores "D" y 584 profesores "P"); opciones de respuesta: $1=$ "Nunca"; $2=$ "Rara vez"; $3=$ "De vez en cuando"; $4=$ "Algunas veces"; $5=$ "Siempre".

En la tabla 2, se visualizan los resultados referentes al estilo de liderazgo transaccional. Al igual que en el caso anterior, las puntuaciones son altas para ambos colectivos; sin embargo, igualmente se han detectado diferencias significativas en todos los componentes relacionados a este liderazgo. Concretamente, el ítem "ofrezco ayuda a los demás a cambio de sus esfuerzos" $\left(\chi^{2}=13,9 / p=, 008\right.$; V de Cramer $\left.=, 14\right)$ y "expreso satisfacción cuando se cumplen las expectativas de los demás" $\left(\chi^{2}=20,9 / p=, 000 ; \mathrm{V}\right.$ de Cramer $=, 17)$ ambos pertenecientes al componente premio por contingencia. Del componente gerencia por excepción pasiva, se han encontrado diferencias en los ítems "espero a que las cosas vayan mal antes de tomar medidas" ( $\chi^{2}=15,9 / p=, 002 ; \mathrm{V}$ de Cramer $\left.=, 15\right)$ y "considero que los problemas deben llegar a ser crónicos para tomar medidas" ( $\chi^{2}=14,9 / p=, 000 ; \mathrm{V}$ de Cramer $\left.=, 18\right)$. En relación con el componente gerencia por excepción activa, se encuentran diferencias en los ítems, "presto atención a las irregularidades, los errores, las excepciones y las desviaciones de la norma" $\left(\chi^{2}=14,2\right.$ / $p=, 005 ; \mathrm{V}$ de Cramer $\left.=, 14\right)$ y "sigo con atención todos los errores" $\left(\chi^{2}=13,8 / p=, 006 ; \mathrm{V}\right.$ de Cramer $\left.=, 13\right)$. 
TABLA 2

Porcentajes y estadísticos de la dimensión liderazgo transaccional

\begin{tabular}{|c|c|c|c|c|c|c|c|c|}
\hline Premio por contingencia & Roll & 1 & 2 & 3 & 4 & 5 & $\mathrm{x}$ & DS \\
\hline \multirow{2}{*}{ 1. Ofrezco ayuda a los demás a cambio de sus esfuerzos. } & $\mathrm{D}$ & 24,8 & 9,6 & 9,6 & 20,0 & 36,0 & 3,33 & 1,62 \\
\hline & $P$ & 19,4 & 8,0 & 12,3 & 26,3 & 34,0 & 3,48 & 1,50 \\
\hline \multirow{2}{*}{$\begin{array}{l}\text { 11. Discuto detenidamente quién es el responsable de alcanzar } \\
\text { los objetivos de rendimiento. }\end{array}$} & $\mathrm{D}$ & 4,0 & 5,6 & 12,0 & 31,2 & 47,2 & 4,12 & 1,08 \\
\hline & $\mathrm{P}$ & 9,4 & 9,9 & 18,7 & 28,4 & 33,6 & 3,67 & 1,28 \\
\hline \multirow{2}{*}{$\begin{array}{l}\text { 16. Dejo muy claro lo que uno puede esperar cuando se alcanzan } \\
\text { los objetivos. }\end{array}$} & $\mathrm{D}$ & 8 & 0 & 4,0 & 23,2 & 72,0 & 4,66 & 0,64 \\
\hline & $P$ & 2,7 & 5,7 & 9,1 & 25,3 & 57,2 & 4,29 & 1,02 \\
\hline \multirow{2}{*}{$\begin{array}{l}\text { 35. Expreso satisfacción cuando se cumplen las expectativas de } \\
\text { los demás. }\end{array}$} & $\mathrm{D}$ & 0 & 0 & 1,6 & 16,0 & 82,4 & 4,81 & 0,43 \\
\hline & $P$ & 2,2 & 5,0 & 8,2 & 19,9 & 64,7 & 4,40 & ,98 \\
\hline \multicolumn{9}{|l|}{ Gerencia por excepción pasiva } \\
\hline \multirow{2}{*}{ 3. No intervengo a no ser que los problemas presenten gravedad. } & $\mathrm{D}$ & 16,0 & 14,4 & 15,2 & 37,6 & 16,8 & 3,25 & 1,34 \\
\hline & $\mathrm{P}$ & 9,8 & 14,9 & 16,0 & 29,8 & 29,5 & 3,54 & 1,31 \\
\hline \multirow{2}{*}{ 12. Espero a que las cosas vayan mal antes de tomar medidas. } & $\mathrm{D}$ & 70,4 & 16,8 & 1,6 & 6,4 & 4,8 & 1,58 & 1,12 \\
\hline & $P$ & 54,0 & 21,0 & 9,3 & 8,9 & 6,8 & 1,94 & 1,26 \\
\hline \multirow{2}{*}{ 17. Creo profundamente en la frase "Si no está roto, no lo arregles". } & $\mathrm{D}$ & 44,0 & 25,6 & 11,2 & 8,8 & 10,4 & 2,16 & 1,35 \\
\hline & $\mathrm{P}$ & 46,7 & 20,3 & 12,5 & 11,9 & 8,6 & 2,15 & 1,34 \\
\hline \multirow{2}{*}{$\begin{array}{l}\text { 20. Considero que los problemas deben llegar a ser crónicos para } \\
\text { tomar medidas. }\end{array}$} & $\mathrm{D}$ & 71,2 & 13,6 & 2,4 & 3,2 & 9,6 & 1,66 & 1,28 \\
\hline & $\mathrm{P}$ & 51,5 & 18,0 & 9,9 & 11,2 & 9,4 & 2,09 & 1,38 \\
\hline \multicolumn{9}{|l|}{ Gerencia por excepción activa } \\
\hline \multirow{2}{*}{$\begin{array}{l}\text { 4. Presto atención a las irregularidades, los errores, las excepciones } \\
\text { y las desviaciones de la norma. }\end{array}$} & $\mathrm{D}$ & 1,6 & 1,6 & 9,6 & 15,2 & 72,0 & 4,54 & 0,86 \\
\hline & $P$ & 3,3 & 6,2 & 12,3 & 23,5 & 54,7 & 4,20 & 1,08 \\
\hline \multirow{2}{*}{$\begin{array}{l}\text { 22. Centro toda mi atención en resolver los errores, las quejas y } \\
\text { los fallos que se producen. }\end{array}$} & $\mathrm{D}$ & 8 & 4,0 & 20,8 & 26,4 & 48,0 & 4,17 & 0,95 \\
\hline & $\mathrm{P}$ & 2,9 & 6,7 & 13,7 & 28,3 & 48,4 & 4,13 & 1,06 \\
\hline \multirow{2}{*}{ 24. Sigo con atención todos los errores. } & $\mathrm{D}$ & 3,2 & 1,6 & 8,8 & 33,6 & 52,8 & 4,31 & 0,94 \\
\hline & $\mathrm{P}$ & 3,6 & 7,9 & 14,8 & 32,4 & 41,3 & 4,00 & 1,09 \\
\hline \multirow{2}{*}{ 27. Dirijo mi atención hacia los casos que no cumplen las normas. } & $\mathrm{D}$ & 7,2 & 6,4 & 19,2 & 31,2 & 36,0 & 3,82 & 1,20 \\
\hline & $\mathrm{P}$ & 7,4 & 8,4 & 19,2 & 26,7 & 38,3 & 3,80 & 1,24 \\
\hline
\end{tabular}

Fuente: Elaboración propia.

Notas: $\mathrm{n}=709$ (125 directores "D" y 584 profesores "P"); opciones de respuesta: $1=$ "Nunca"; $2=$ "Rara vez"; $3=$ "De vez en cuando"; $4=$ "Algunas veces"; y $5=$ "Siempre".

En los estilos de liderazgo transformacional y transaccional, se observa como las personas directoras obtienen puntuaciones medias superiores. Sin embargo, para el estilo de liderazgo laissez-faire la población directora tienen promedios inferiores al colectivo docente en todos los ítems. Aunque cabe resaltar las bajas puntuaciones para este estilo, se han hallado diferencias significativas en tres de los cuatro ítems que componen este factor: "me ausento cuando se me necesita" $\left(\chi^{2}=34,1 / p=, 000 ; \mathrm{V}\right.$ de Cramer $\left.=, 22\right)$; "evito tomar decisiones $\left(\chi^{2}=21,2 / p=, 000 ; \mathrm{V}\right.$ de Cramer $\left.=, 17\right)$ y "me retraso en dar respuesta a cuestiones urgentes" $\left(\chi^{2}=9,7 / p=, 035 ; \mathrm{V}\right.$ de Cramer $\left.=, 11\right)$. 
TABLA 3

Porcentajes y estadísticos de la dimensión liderazgo laissez-faire

\begin{tabular}{|c|c|c|c|c|c|c|c|c|}
\hline Laissez-faire & Roll & 1 & 2 & 3 & 4 & 5 & $\mathrm{X}$ & DS \\
\hline \multirow{2}{*}{$\begin{array}{l}\text { 5. Evito involucrarme cuando surgen cuestiones de } \\
\text { importancia. }\end{array}$} & $\mathrm{D}$ & 53,6 & 14,4 & 8,8 & 14,4 & 8,8 & 2,10 & 1,41 \\
\hline & $\mathrm{P}$ & 43,0 & 17,1 & 12,5 & 17,0 & 10,4 & 2,35 & 1,43 \\
\hline \multirow{2}{*}{ 7. Me ausento cuando se me necesita. } & $\mathrm{D}$ & 72,8 & 8,8 & 3,2 & 5,6 & 9,6 & 1,70 & 1,33 \\
\hline & $\mathrm{P}$ & 47,7 & 21,3 & 11,1 & 13,4 & 6,5 & 2,10 & 1,30 \\
\hline \multirow{2}{*}{ 28. Evito tomar decisiones. } & $\mathrm{D}$ & 72,0 & 15,2 & 1,6 & 8,0 & 3,2 & 1,55 & 1,07 \\
\hline & $\mathrm{P}$ & 51,7 & 18,3 & 8,9 & 12,2 & 8,9 & 2,08 & 1,37 \\
\hline \multirow{2}{*}{ 33. Me retraso en dar respuesta a cuestiones urgentes. } & $D$ & 36,8 & 31,2 & 9,6 & 16,8 & 5,6 & 2,23 & 1,26 \\
\hline & $P$ & 35,8 & 20,4 & 12,7 & 20,4 & 10,7 & 2,50 & 1,42 \\
\hline
\end{tabular}

Fuente: Elaboración propia.

Notas: $n=709$ (125 directores "D" y 584 profesores "P"); opciones de respuesta: $1=$ "Nunca"; $2=$ "Rara vez"; $3=$ "De vez en cuando"; $4=$ "Algunas veces"; 5 = "Siempre".

Como ya se ha indicado anteriormente, la escala mide, además de los estilos de liderazgo, tres subescalas. En líneas generales (tabla 4) las directoras y los directores obtienen puntuaciones más elevadas en las tres subescalas (efectividad, esfuerzo extra y satisfacción) que las halladas por el colectivo de docentes con el cual laboran. En el caso de la efectividad, se han encontrado diferencias significativas en los cuatro ítems: "soy eficaz en satisfacer las necesidades de índole laboral de los demás" ( $\chi^{2}=16,1 / p=, 003 ; \mathrm{V}$ de Cramer = ,15); "soy eficaz al representar a mi grupo ante autoridades superiores" $\left(\chi^{2}=20,2 / p=, 000\right.$; $V$ de Cramer = ,17); "soy eficaz en satisfacer los requisitos del centro educativo" $\left(\chi^{2}=14,0 / p=, 007 ; \mathrm{V}\right.$ de Cramer $=, 14)$ y "lidero un grupo que es eficaz" $\left(\chi^{2}=15.8, p=, 003 ; \mathrm{V}\right.$ de Cramer $\left.=, 15\right)$. En el caso de la subescala esfuerzo extra, se obtienen diferencias en los ítems "realzo en los demás el deseo de triunfar" $\left(\chi^{2}=27,7 /\right.$ $p=, 000 ; \mathrm{V}$ de Cramer $=, 20)$ y "aumento la voluntad de los demás para poner el máximo desempeño" ( $\chi^{2}$ $=13,3 / p=, 010 ; V$ de Cramer $=, 14)$. Igualmente, para la última subescala de satisfacción se encuentran diferencias en los dos ítems que la componen: "utilizo métodos de liderazgo agradable" $\left(\chi^{2}=13,5 / p=\right.$ $, 009 ; \mathrm{V}$ de Cramer $=, 14)$ y "trabajo satisfactoriamente con los demás" $\left(\chi^{2}=18,8\right.$ / $p=, 001$; V de Cramer = ,16). 
TABLA 4

Porcentajes y estadísticos de las subescalas efectividad, esfuerzo extra y satisfacción

\begin{tabular}{|c|c|c|c|c|c|c|c|c|}
\hline Efectividad & Roll & 1 & 2 & 3 & 4 & 5 & $x$ & DS \\
\hline \multirow{2}{*}{$\begin{array}{l}\text { 37. Soy eficaz en satisfacer las necesidades de índole laboral } \\
\text { de los demás. }\end{array}$} & D & 0 & 0 & 5,6 & 32,8 & 61,6 & 4,56 & 0,60 \\
\hline & $P$ & 1,2 & 5,3 & 13,5 & 27,2 & 52,8 & 4,25 & ,96 \\
\hline \multirow{2}{*}{$\begin{array}{l}\text { 40. Soy eficaz al representar a mi grupo ante autoridades } \\
\text { superiores. }\end{array}$} & D & 0 & 0 & 3,2 & 20,0 & 76,8 & 4,74 & 0,51 \\
\hline & $\mathrm{P}$ & 1,9 & 3,6 & 11,3 & 25,0 & 58,2 & 4,34 & 94 \\
\hline \multirow{2}{*}{$\begin{array}{l}\text { 43. Soy eficaz en satisfacer los requisitos del centro } \\
\text { educativo. }\end{array}$} & $\mathrm{D}$ & 0 & 0 & 2,4 & 25,0 & 72,6 & 4,70 & 0,51 \\
\hline & $P$ & 1,7 & 3,4 & 9,1 & 23,8 & 62,0 & 4,41 & 91 \\
\hline \multirow{2}{*}{ 45. Lidero un grupo que es eficaz. } & $\mathrm{D}$ & 8 & 8 & 2,4 & 29,6 & 66,4 & 4,60 & 0,66 \\
\hline & $P$ & 1,9 & 6,2 & 10,1 & 25,2 & 56,6 & 4,28 & 1,00 \\
\hline \multicolumn{9}{|l|}{ Esfuerzo extra } \\
\hline \multirow{2}{*}{$\begin{array}{l}\text { 39. Hago que los demás hagan más de lo que esperaban } \\
\text { hacer. }\end{array}$} & $\mathrm{D}$ & 3,2 & 6,4 & 12,0 & 36,8 & 41,6 & 4,07 & 1,04 \\
\hline & $\mathrm{P}$ & 7,5 & 12,2 & 13,0 & 32,5 & 34,8 & 3,75 & 1,25 \\
\hline \multirow{2}{*}{ 42. Realzo en los demás el deseo de triunfar. } & $\mathrm{D}$ & 8 & 8 & 0 & 15,2 & 83,2 & 4,79 & 0,56 \\
\hline & $P$ & 3,4 & 5,3 & 8,2 & 22,3 & 60,8 & 4,32 & 1,05 \\
\hline \multirow{2}{*}{$\begin{array}{l}\text { 44. Aumento la voluntad de los demás para poner el máxi- } \\
\text { mo desempeño. }\end{array}$} & $\mathrm{D}$ & 1,6 & 0 & 2,4 & 25,6 & 70,4 & 4,63 & 0,69 \\
\hline & $P$ & 3,3 & 4,3 & 7,5 & 27,2 & 57,7 & 4,32 & 1,00 \\
\hline \multicolumn{9}{|l|}{ Satisfacción } \\
\hline \multirow{2}{*}{ 38. Utilizo métodos de liderazgo agradable. } & $\mathrm{D}$ & 0 & 0 & 8,8 & 21,6 & 69,6 & 4,61 & 0,65 \\
\hline & $P$ & 2,9 & 4,5 & 8,4 & 27,5 & 56,7 & 4,31 & 1,00 \\
\hline \multirow{2}{*}{ 41. Trabajo satisfactoriamente con los demás. } & $\mathrm{D}$ & 0 & 0 & 2,4 & 17,6 & 80,0 & 4,78 & 0,47 \\
\hline & $\mathrm{P}$ & 1,7 & 3,4 & 9,2 & 23,3 & 62,4 & 4,41 & 91 \\
\hline
\end{tabular}

Fuente: Elaboración propia.

Notas: $n=709$ (125 directores "D" y 584 profesores "P"); opciones de respuesta: $1=$ "Nunca"; $2=$ "Rara vez"; $3=$ "De vez en cuando"; $4=$ "Algunas veces"; y $5=$ "Siempre".

Para la comprobación de la normalidad se realizó la prueba de Kolmogorov-Smirnov, obteniendo un nivel " $p$ " no significativo $(p>, 05)$ para ambos grupos (directores y docentes). Seguidamente se llevó a cabo la prueba de Levene para la comprobación de la homogeneidad de varianzas. Posteriormente, una vez cumplidos los supuestos anteriores, se realizó la prueba t de Student para la igualdad de medias (tabla $5)$, se encontraron diferencias significativas $(p=, 000)$ entre colectivos en las puntuaciones en todos los estilos, y se obtuvieron medias superiores en las personas directoras que en el colectivo docente, a excepción del estilo laissez-faire en el cual los profesionales en docencia obtienen puntuaciones superiores. 
TABLA 5

Estadísticos de grupo y resultados de las pruebas $t$ de Student para cada estilo de liderazgo

\begin{tabular}{|c|c|c|c|c|c|c|}
\hline \multirow{2}{*}{$\begin{array}{l}\text { Estilo de } \\
\text { liderazgo }\end{array}$} & \multicolumn{3}{|c|}{ Estadísticos de grupo } & \multicolumn{3}{|c|}{ Prueba T para la igualdad de medias } \\
\hline & Rol & Media & DS & $\mathrm{t}$ & gl & Sig. (bilateral) \\
\hline \multirow{2}{*}{ Transformacional } & Directores & 4,27 & ,37 & \multirow{2}{*}{3,42} & \multirow{2}{*}{707} & \multirow{2}{*}{001} \\
\hline & Profesores & 4,05 & ,67 & & & \\
\hline \multirow{2}{*}{ Transaccional } & Directores & 4,09 &, 43 & \multirow{2}{*}{4,25} & \multirow{2}{*}{707} & \multirow{2}{*}{,000 } \\
\hline & Profesores & 3,85 &, 59 & & & \\
\hline \multirow{2}{*}{ Laissez-faire } & Directores & 1,90 &, 82 & \multirow{2}{*}{4,12} & \multirow{2}{*}{707} & \multirow{2}{*}{, 000} \\
\hline & Profesores & 2,25 & 1,01 & & & \\
\hline \multirow{2}{*}{ Efectividad } & Directores & 4,64 & ,39 & \multirow{2}{*}{4,41} & \multirow{2}{*}{707} & \multirow{2}{*}{, 000} \\
\hline & Profesores & 4,32 & 81 & & & \\
\hline \multirow{2}{*}{ Satisfacción } & Directores & 4,69 & ,46 & \multirow{2}{*}{4,10} & \multirow{2}{*}{707} & \multirow{2}{*}{, 000} \\
\hline & Profesores & 4,35 &, 89 & & & \\
\hline \multirow{2}{*}{ Esfuerzo extra } & Directores & 4,50 &, 56 & \multirow{2}{*}{4,48} & \multirow{2}{*}{707} & \multirow{2}{*}{, 000} \\
\hline & Profesores & 4,12 & 89 & & & \\
\hline
\end{tabular}

Fuente: Elaboración propia.

\section{DISCUSIÓN DE RESULTADOS}

Los resultados muestran puntuaciones altas, tanto en la autopercepción de las personas directoras como del personal docente en los estilos de liderazgo transformacional y transaccional, obteniendo bajas puntuaciones en el estilo laissez-faire, aspectos positivos para las organizaciones educativas.

Específicamente, el liderazgo transformacional (tabla 1) es valorado en todos sus componentes con puntuaciones muy altas por las personas directoras, sin embargo, las evaluaciones de las personas docentes a pesar de ser altas denotan puntuaciones inferiores en la escala en relación con la percepción del personal directivo. Es importante destacar que, aunque no sean valoraciones polarizadas, los resultados dejan entrever diferencias significativas de percepción, aspecto que repercute en la dinámica organizacional caracterizada por el trabajo colaborativo para el logro de metas (García et al., 2018).

La estimulación intelectual es percibida con mejores valoraciones desde la autopercepción de las personas directoras en el ejercicio de sus funciones que la asignada por las personas docentes. Esta condición se presenta en todos los ítems del componente en cuestión, y tiene relación directa con la forma en como los miembros del centro educativo resuelven de manera creativa e innovadora los problemas que enfrentan, guiados por la persona directora (López, 2017). Así mismo, la influencia idealizada, tanto por conducta como por atributos, es valorada de manera similar a la variable anterior. En este componente, se alejan las percepciones de las personas docentes respecto a la de las directivas, en aspectos que valoran una revisión crítica de las asunciones con que se direcciona el centro educativo o el enfocar los problemas desde diferentes ángulos, condición que plantea miradas distintas de una misma realidad y que puede repercutir en los valores que se comparten en el centro educativo, (López, 2017). En esta misma dirección llama la atención que el único ítem donde tienen una mayor valoración el personal docente es el que refiere a hacer ostentación de su poder y confianza, lo que apunta a un elemento negativo que puede asociarse con credibilidad y confianza (Castro, 2015).

En cuanto a la variable de motivación por inspiración se repite una autopercepción mejor valorada por las personas directoras en relación con el colectivo docente. Este aspecto se asocia al optimismo y visión de 
futuro que impregna la persona que lidera en su equipo de trabajo. Situación que puede repercutir de forma negativa en el rendimiento efectivo del equipo en dirección de objetivos y metas comunes (Hoy y Miskel, 2008).

El mismo comportamiento presenta la variable de consideraciones individuales, la cual muestra valores más altos en las personas directoras que los obtenidos desde la percepción docente. La misma se vincula a la necesidad que tienen las personas de sentirse valorados como individuos y capaces de desarrollar sus propias potencialidades. Lo anterior contribuye a una sensación de bienestar individual que repercute en el ambiente del centro educativo. (Perilla, 2015).

En cuanto al liderazgo transaccional (tabla 2), se observa que es el segundo estilo de liderazgo en orden de predominancia por ambos colectivos. La variable premio por contingencia, en términos generales, mantiene la misma percepción casi en todos los ítems valorados; las personas directoras se evalúan con autopercepciones más altas que las personas docentes. Sin embargo, el ítem de ofrecer ayuda a los demás a cambio de sus esfuerzos es el único que las personas docentes evalúan más alto que las directivas. La percepción del cuerpo docente es que el cuerpo directivo les apoya o ayuda por su esfuerzo o desempeño en el centro educativo. Queda explícita una percepción entre las personas docentes que también es reconocida por las personas directoras en sus prácticas de liderazgo: el reconocimiento y apoyo hacia las personas del equipo de trabajo vinculadas con el cumplimiento de expectativas y de objetivos fijados por la persona directora (Brito, 2016).

En este mismo estilo transaccional, la variable gerencia por excepción pasiva muestra resultados muy distintos entre los colectivos. La mayoría de los ítems son valorados más altos por las personas docentes en relación con las personas directivas. Esta variable refiere a la no intervención en los problemas que se presentan en el centro educativo hasta que sean de gravedad, a situaciones problemáticas que se vuelven "crónicas" para que los directivos presten atención, lo que refleja un punto negativo desde la percepción de las personas docentes en relación con las prácticas de liderazgo de los directivos, coincidiendo con Brito, (2016).

En cuanto a la variable de gerencia por excepción activa, se reitera el mismo comportamiento de percepción, en donde las personas directoras se valoran con mayores puntajes que las personas docentes. Desde la autopercepción de los directivos, las prácticas de liderazgo se dirigen hacia el cumplimiento de normas, atención de los errores y seguimiento de faltas por parte de las personas docentes, lo cual concuerda con lo mencionado por Orellana (2019).

En cuanto al estilo de laissez-faire (Tabla 3), cabe destacar que la valoración de las personas docentes es más alta en todos los ítems, que las personas directoras, es decir que consideran en mayor medida la existencia de este estilo en los centros educativos. Estos resultados muestran que algunas personas directivas son conscientes de que no se involucran en los procesos que demanda el centro educativo, ausentándose cuando son requeridos, evitando tomar decisiones y en algunos casos retrasando las respuestas de temas urgentes. No se justifican desde las teorías de liderazgo este tipo de prácticas, ya que este comportamiento por parte de la persona directora trae consecuencias no deseadas en la cultura organizacional, en donde sus miembros asumen de manera aislada la búsqueda de alternativas para los problemas que les aquejan, repercutiendo en la calidad de la oferta educativa (Zuzama, 2015).

Finalmente, se presentan las tres variables independientes (tabla 4), que no se asocian a ningún estilo de liderazgo en específico; efectividad, esfuerzo extra y satisfacción. En estas variables se observa que las personas directoras se valoran con puntuaciones más altas en todos los ítems que las personas docentes, pues consideran que estimulan a su equipo para alcanzar los objetivos que se plantean (Bracho, y García, 2013). Igualmente, alientan a las personas docentes para asumir tareas extras, favoreciendo un clima organizacional saludable en donde todos los miembros del centro educativo se sienten satisfechos (García et al., 2015). 


\section{CONCLUSIONES}

El estilo de liderazgo predominante en las personas que gestionan los centros educativos de la Dirección Regional de Educación de Heredia es el transformacional. Lo que supone un aspecto positivo ya que se direcciona a un funcionamiento efectivo, cumpliendo con altas expectativas de la comunidad educativa.

Otro estilo de liderazgo predominante es el transaccional, el cual se basa en intercambios entre el líder y su equipo, que favorece capacidades individuales para aportar de manera eficiente al logro de los objetivos planteados en el centro educativo.

Se evidencia como el estilo laissez-faire, aunque en menor medida que los anteriores, está presente en los centros educativos. Cabe destacar, que el estilo presume de ausencia de autoridad y participación oportuna para la búsqueda de soluciones a los problemas de la comunidad educativa.

Se observan diferencias significativas en los tres estilos mencionados. Los directores tienen una percepción mayor que el colectivo docente en el ejercicio de prácticas que direccionan al estilo transformacional y transaccional. Por el contrario, los docentes consideran que se ejerce un estilo laissez-faire en mayor medida que el personal directivo.

La efectividad, el esfuerzo extra y la satisfacción se favorecen de manera intencionada según la valoración de ambos colectivos. Se visualizan esfuerzos por parte del personal directivo para el logro de objetivos, el estímulo para el desarrollo de las capacidades individuales y los ambientes laborales que permiten potenciar equipos de alto rendimiento.

Aunque los resultados son óptimos en el sentido de un liderazgo transformacional predominante, se recomienda a los equipos de directivos, docentes y autoridades fortalecer de forma consciente e intencionada este estilo de liderazgo para la consecución de metas colaborativas.

El liderazgo transformacional surge como uno de los estilos que favorece un trabajo colaborativo en las instituciones educativas. En este sentido, se sugiere como línea de investigación futura, el abordaje desde un enfoque cualitativo, que permita profundizar en las prácticas exitosas de gestión educativa desde este tipo de liderazgo. Por otro lado, y al encontrar diferencias significativas en las percepciones del estilo de liderazgo entre los colectivos participantes, se hacen necesarios estudios que permitan establecer acciones estratégicas en la comunidad educativa hacia metas comunes y formación continua en el área de liderazgo pedagógico.

\section{AGRADECIMIENTO}

Este artículo surge en el marco de la investigación denominada el clima organizacional y la relación con los estilos de liderazgo en centros educativos costarricenses (Código 0225-18), desarrollada en la carrera de Administración Educativa en la División de Educación para el Trabajo (CIDE) de la Universidad Nacional.

\section{REFERENCIAS}

Arnal, J., Rincón, D. y Latorre, A. (1992). Investigación educativa: fundamentos y metodología. Barcelona, España, Ed. Labor.

Ayoub, J.L. (2011). Estilos de liderazgo y su eficacia en la administración pública mexicana. Metodología y resultados de una investigación empírica. North Carolina, Luluenterprises, Inc. 
Barrios, S. A. (2020). Estado del arte del liderazgo transformacional en la educación universitaria. Revista Científica Conecta Libertad, 4(1), 75-81. Recuperado de: http://revistaitsl.itslibertad.edu.ec/index. php/ITSL/article/view/114/328

Bass, B. \& Avolio, B. (Eds.). (1994). Improving organizational effectiveness through transformational leadership. California, Sage.

Bolívar, A. (2015). Un liderazgo pedagógico en una comunidad que aprende. España: Universidad de Granada. Revista Padres y Maestros, 361, 23-27. Recuperado de: https://www.researchgate.net/ publication/322298404_Liderazgo_pedagogico

Bracho, O., García, J. (2013). Algunas consideraciones teóricas sobre el liderazgo transformacional. Revista de Estudios Interdisciplinarios en Ciencias Sociales, 15 (2), 165-177. Recuperado de https://dialnet. unirioja.es/descarga/articulo/4451074.pdf

Brito, J. (2016). Personalidad y Liderazgo. INNOVA Research Journal, 1(3), 76-83. Guayaquil. Ecuador. Recuperado de https://dialnet.unirioja.es/descarga/articulo/6183809.pdf

Campos, A. y Campos, L. (2014). Optimizando la gestión y administración profesionales. Comunicación y liderazgo laissez faire enfermero. España: Revista Española de Comunicación en Salud, 6(1), 85-93. Recuperado de: http://www.aecs.es/6_1_8.pdf

Carreño, I. (2020). Liderazgo distribuido en las organizaciones educativas madrileñas: un estudio de casos. Revista Ibero-Americana de Estudos em Educação, 16(1). https://doi.org/10.21723/riaee.v16i1.13423

Castro, J. (2015). Dimensiones del liderazgo transformacional predominantes en la directora de la Institución Educativa Privada Federico Villarreal de la provincia de Talara. (Tesis de Maestría en Educación con Mención en Gestión Educativa no publicada). Universidad de Piura. Facultad de Ciencias de la Educación. Piura, Perú.

Cerdas, V., García, J. A., Torres, N., y Fallas, M. A. (2017). Análisis de la gestión administrativa de centros educativos costarricenses: Percepción del colectivo docente y la dirección. Revista Ensayos Pedagógicos, 12(2), 95-122. https://doi.org/10.15359/rep.12-2.5

Contreras, T. (2016). Liderazgo pedagógico, liderazgo docente y su papel en la mejora de la escuela: una aproximación teórica. Revista: Propósitos y Representaciones, 4(2), 231-284. http://dx.doi.org/10.20511/ pyr2016.v4n2.123

Dean, M., Rushton, G., Edwards, A. \& Jarrell, V. (2016). Tracking the Growth of Teacher Leaders Using the Multifactor Leadership Questionnaire (MLQ) and Teacher Self-Efficacy Instruments. Conference: National Association for Research in Science Teaching Annual International Conference, At Baltimore, MD. Recuperado de https://bit.ly/3ijv6iA

Duitama, G. (2017). El liderazgo transformacional como una opción de crecimiento para los empresarios. Colombia: Libros Universidad Nacional Abierta y a Distancia.

Espinoza, E. y Elgoibar, P. (2019). Patrones de liderazgo en los administradores ecuatorianos: Impacto del género y la educación. Global Conference on Business and Finance Proceedings, 14(2), 383-393. Recuperado de: https://bit.ly/30Cky8d

Fernández, C. y Quintero, N. (2017). Liderazgo transformacional y transaccional en emprendedores venezolanos. Revista Venezolana de Gerencia, 22(77), 56-74. Recuperado de: https://www.redalyc. org/pdf/290/29051457005.pdf

Flores, C. y Homrani, M. (2015). Liderazgo transformacional. Equipos directivos en centros educativos de alto rendimiento escolar. España: Universidad de Granada. Journal for Educators, Teachers and Trainers, 6(1), 220-235. Recuperado de: http://jett.labosfor.com/index.php/jett/article/view/137/220

Gajardo, J. y Ulloa, J. (2016). Liderazgo Pedagógico, Conceptos y Tensiones. Nota Técnica N. `6, Líderes Educativos, Centro de Liderazgo para la Mejora Escolar: Universidad de Concepción, Chile. 
García, J. A., y Cerdas, V. (2019). Estilos de liderazgo de los directivos escolares costarricenses: transformando las organizaciones educativas. Innovaciones educativas, 21(31), 5-21. https://doi.org/10.22458/ ie.v21i31.2690

García, J. A., Cerdas, V., y Torres, N. (2018). Gestión curricular en centros educativos costarricenses: Un análisis desde la percepción docente y la dirección. Revista Electrónica Educare, 22(1), 225-252. http://dx.doi.org/10.15359/ree.22-1.11

García, Y., Mendoza, I., y Ruíz, S. (2015). Liderazgo y su relación con las variables de resultado en una empresa productora de envases de plástico. Revista de Administración y Finanzas, 2(3), 454-469. Hidalgo. México. Recuperado de: https://bit.ly/3kmuUkq

Gil, A., Muñíz, M. y Delgado, A. (2008). El liderazgo transformativo en el ámbito escolar: Un esfuerzo de investigación en acción y cooperación entre instituciones de Educación Superior. Revista Universitaria de Investigación, 1, 13-33. Recuperado de: https://dialnet.unirioja.es/servlet/articulo?codigo=2781916

Hauserman, C. \& Stick, S. (2013). The Leadership Teachers Want from Principals: Transformational. United States of America: Athabasca University \& University of Nebraska-Lincoln. Canadian Journal of Education 36(3) ,184-203. Recuperado de: https://files.eric.ed.gov/fulltext/EJ1057940.pdf

Hernández, R., Fernández, C. y Baptista, P. (2010). Metodología de la investigación. México D.F., México: Editorial Mc Graw Hill.

Hoy, W. \& Miskel, C. (2008). Educational Administration: theory, research and practice. New York. United States. Mc Graw Hill.

Hurtado, J. (2019). Un acercamiento sintagmático al concepto de liderazgo. Desarrollo Gerencial, 11(1), 190-208. https://doi.org/10.17081/dege.11.1.3465

López, E. (2017). Dimensiones del liderazgo transformacional y capacidad de aprendizaje organizacional en Pymes. Espacios 38(57) 1-16. Recuperado de: http://www.revistaespacios.com/ a17v38n57/17385716.html

Orellana, K. (2019). El liderazgo del director y el desempeño docente autopercibido en un grupo de colegios privados salvadoreños. Revista Internacional de Estudios en Educación, 19(1), 47-63. Recuperado de: https://riee.um.edu.mx/index.php/RIEE/article/view/206/193

Pacsi, A., Estrada, W., Pérez, A. y Cruz, P. (2015). Liderazgo Laissez Faire. Revista Cuaderno Empresarial, 1(1), 9-16. Recuperado de: https://bit.ly/2DGr7O7

Perilla, L. (2015). Relación del liderazgo transformacional con la salud y el bienestar del empleado: el rol mediador de la confianza en el líder. (Tesis de doctorado). Bogotá. Colombia. Recuperado de: https:// bit.ly/3gD1PPn

Perles, G., y Santiago, M. (2000). Ética y liderazgo empresarial: una complementariedad necesaria. Papeles de ética, economía y dirección, 5, 1-14. Recuperado de: https://bit.ly/3ifzb7B

Prieto, M., Contreras, F. y Espinosa, J. (2019). Liderazgo y comportamiento innovador del trabajador en personal administrativo de una institución educativa. Revista Diversitas: Perspectivas en Psicología, 16(1), 25-35. Recuperado de: https://revistas.usantotomas.edu.co/index.php/diversitas/article/ view/5540/5456

Rodríguez, G. (2011). Funciones y rasgos del liderazgo pedagógico en los centros de enseñanza. Revista: Educación y Educadores, 14(2), 253-267. Recuperado de: https://www.redalyc.org/ pdf/834/83421404003.pdf

Sánchez, A. V. (2015). Importancia e impacto del liderazgo educativo. Padres y Maestros/Journal of Parents and Teachers, (361), 6-11. Recuperado de: http://revistas.upcomillas.es/index.php/padresymaestros/ article/view/5335 
Sharma, S. (2020). Examen de las propiedades psicométricas del cuestionario de liderazgo multifactorial y el cuestionario de desempeño laboral individual en el sector bancario indio. Estudios en topónimos indios, 40(1), 237-249.

Torrecilla, F. J. (2016). La investigación sobre liderazgo educativo: una mirada desde el presente, proyectada al futuro. Revista Fuentes, (14), 9-14. Recuperado de: https://repositorio.uam.es/bitstream/ handle/10486/661175/Investigacion_Murillo_RF_2013.pdf?sequence=1

Vílchez, J., Alberola, E., Monte, P. R., y Ferraz, H. (2019). Relación entre los estilos de liderazgo Transformacional y laissez-faire y el Síndrome de Quemarse por el Trabajo en profesores de educación secundaria. Acciones e investigaciones sociales, (39), 223-254. Recuperado de: https://dialnet. unirioja.es/servlet/articulo?codigo $=6728778$

Villa, A. (2019). Liderazgo: una clave para la innovación y el cambio educativo. España: Universidad de Deusto. Revista de Investigación Educativa, 37(2), 301-326. Recuperado de: https://revistas.um.es/ rie/article/view/365461/266091

Yang, I. (2015). Positive effects of laissez-faire leadership: Conceptual exploration. The Journal of Management Development, 34(10), 1246-1261. Recuperado de: https://doi.org/10.1108/ JMD-02-2015-0016

Zuzama, J. (2015). Liderazgo: estilos de liderazgo según Kurt Lewin y análisis de un caso real. Mallorca, España: Universitat de les Illes Balears. 